\title{
Transverse and longitudinal crushing of aluminum-foam filled tubes
}

\author{
I.W. Hall $^{\mathrm{a}, *}$, M. Guden ${ }^{\mathrm{b}}$, T.D. Claar ${ }^{\mathrm{c}}$ \\ a Department of Mechanical Engineering, 126 Spencer Laboratory, University of Delaware, Newark, DE 19716-3619, USA \\ ${ }^{\mathrm{b}}$ Department of Mechanical Engineering, Izmir Institute of Technology, Izmir, Turkey \\ ${ }^{\mathrm{c}}$ Fraunhofer Center for Manufacturing and Advanced Materials, Newark, DE 19716, USA
}

Received 21 September 2001; received in revised form 7 January 2002

\begin{abstract}
Al-foam filled and empty tubes of aluminum, brass and titanium were compression tested laterally. The specific energy absorption in filled tubes increased greatly in terms of percentages, and was greatest in aluminum tubes. In transversely tested tubes the foam deformed laterally showing a capability of spreading the deformation. ( $) 2002$ Acta Materialia Inc. Published by Elsevier Science Ltd. All rights reserved.
\end{abstract}

Keywords: Aluminum; Foams; Mechanical properties; Dynamic phenomena

\section{Introduction}

Developments in the understanding and application of aluminum foams have opened the way for their use in a variety of applications including foam-core stiffening, blast-protection, and insulation [1-3]. Also, tubes filled with aluminum foam have recently been investigated for their energy absorption characteristics, particularly in connection with improving the crashworthiness of new generations of vehicles [4-6]. It is found that the buckling characteristics of aluminum foam filled tubes are altered in several significant respects: most notable are that the buckling length of side walls is decreased and the total energy absorbed by

\footnotetext{
${ }^{*}$ Corresponding author. Tel.: +1-302-831-2062; fax: +1-302831-4545.

E-mail address: hall@me.udel.edu (I.W. Hall).
}

the foam/tube combination is greater than the sum of the energy absorbed by the foam (alone) + tube (alone) [4,7]. A commonly observed feature of such axial crushing, using foam either as unsupported cylinders or as foam-filling, is that almost no transverse strain occurs. Consequently, the foam has a Poisson's ratio close to zero, and the walls of tubes pull away from the foam-filling to form empty 'ears'. While this is not necessarily a handicap for blast-protection applications where distributed loading occurs, the inability of foams to transmit strains laterally severely limits the efficiency of energy absorption in large foam structures when loaded by pointed or blunt objects. It would be useful if a large foam volume, struck by a blunt object, could also distribute the strain laterally instead of simply densifying ahead of the indenter. Following recent work in which a series of tests were performed in the axial direction $[5,6]$, the present tests were conducted on other 
geometries in order to characterize the response of foam-filled tubes in the transverse direction. Since these applications are generally concerned with rather high strain rates and prior testing to investigate the force/displacement characteristics was done using the split Hopkinson pressure bar (SHPB), the same technique has been extensively used in the present experiments: however, quasistatic testing has also been performed and indicates that the results are not strain-rate dependent.

\section{Experimental}

In order to investigate effects in materials other than aluminum, additional materials used in these experiments were brass and titanium. The drawn brass tubes $(10 \% \mathrm{Zn})$ were $15.88 \mathrm{~mm}$ in diameter with a wall thickness of $0.81 \mathrm{~mm}$, while the welded titanium tubes (Grade 2) were $12.7 \mathrm{~mm}$ in diameter with a wall thickness of $0.9 \mathrm{~mm}$. Aluminum tubes (3003-H14) were $15.88 \mathrm{~mm}$ in diameter with a wall thickness of $0.85 \mathrm{~mm}$.

Two approaches were taken to filling the tubes with foam. In the first method, tubes were filled by inserting rods of foamable material into the tubes and then introducing them into a furnace at approximately $670{ }^{\circ} \mathrm{C}$ for several minutes to promote foaming as described elsewhere [8]. Some of these tubes were also given an internal surface treatment with a brazing flux to improve adhesion between the foam and the walls of the tube since it has been suggested that this may improve the mechanical properties. Cut-up tests on these samples showed that adhesion was indeed improved and that, after prying out the foam core, some torn ligaments remained in place on the tube wall. Still other tubes were not filled but, in order to compare the properties of tubes with similar mechanical properties, were merely given a heat treatment corresponding to that received by the foam filled tubes. The second approach was to prepare cylindrical cores of foam from a bulk foam plate and press them into the tubes. This second method (a) preserved the initial mechanical properties of the tubes and (b) clearly avoided any adhesion between the foam and tube wall. The average density of the foam was about $0.4 \mathrm{~g} \mathrm{~cm}^{-3}$ and detailed information on mechanical properties is given elsewhere [9].

Sample tubes of each alloy were also tested in the untreated, or as-received, condition. Consequently four different types of samples were tested and they will be referred to below as follows: (a) AR, denoting as-received tube, no treatment; (b) F1, denoting a tube that was foam-filled by thermal route; (c) F2, denoting a tube that was foamfilled by mechanical means; (d) HT, denoting an empty tube given a heat treatment corresponding to that of foam-filling by the thermal route. The tubes were then machined so that their top and bottom faces were flat, parallel and normal to the longitudinal axis. Tests were performed in the longitudinal and transverse directions. Quasi-static compression tests were carried out on an Instron machine with a cross-head displacement rate of $0.02 \mathrm{~mm} \mathrm{~s}^{-1}$. Dynamic compression tests were carried out using the SHPB according to methods described previously [8]. Obviously the generation of stress/strain curves for these samples from SHPB data is highly problematical, both for the metal foams and the transversely crushed tubes. Consequently, the approach adopted here, as elsewhere [8], has been to describe the deformation simply in terms of force and displacement. In order to determine whether there is actually any advantage in filling the tubes with foam, the forcedisplacement data have also been converted into specific energy using the relation in [4]. Transverse samples were $15 \mathrm{~mm}$ in length so that they were uniformly compressed by the $19 \mathrm{~mm}$ diameter bars of the SHPB. Most of the longitudinal specimens were also $\sim 15 \mathrm{~mm}$ long, although a few longer ones were tested to investigate any possible effect of sample length on the results: none was noted.

\section{Results}

Since quasi-static and dynamic tests on empty and filled tubes in the axial direction confirmed previous results showing the effectiveness of aluminum foam in increasing the stress necessary to crush the tubes [8], principally transverse data will be presented here. Fig. 1(a) shows quasi-static data for an AR titanium tube, a cylinder of foam (of 

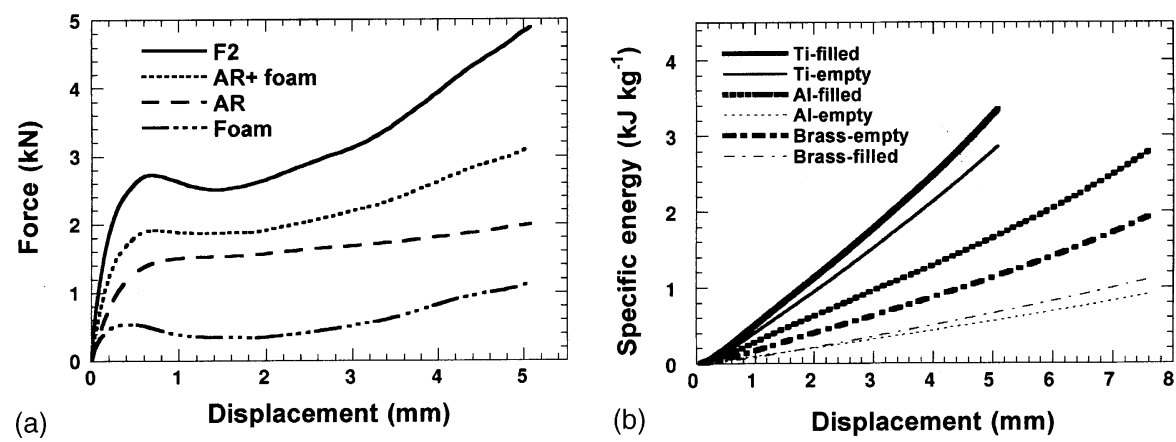

Fig. 1. (a) Force vs. displacement data for a series of transversely tested Ti tubes. (b) Typical specific energy vs. displacement curves for each alloy.

dimensions such that it would just 'fit' into the tube), the algebraic sum of these two and, finally, an F2 foam-filled titanium tube. It is clearly seen that the force measured on the foam-filled tube is considerably greater than the simple sum of the two components. Even on a specific energy basis, Fig. 1(b), there is still considerable advantage in the foam-filling for all the alloys. The increases in specific energy for deformation corresponding to $25 \%$ reduction in diameter for $\mathrm{Ti}$, brass and $\mathrm{Al}$ tubes, respectively, are approximately $0.28,0.3$ and $0.75 \mathrm{~kJ} \mathrm{~kg}^{-1}$.

Turning to the dynamic data, Fig. 2(a) shows typical dynamic force vs. displacement data for axially loaded brass tube samples. Although only relatively small deformations are presented here it is again seen that filled brass tubes offer an increased energy absorption over empty tubes of similar yield/flow strength (Fig. 2(b)). However, the data of Fig. 2(a) and (b) also show that, although the surface treatment was seen to improve adhesion between tube and foam (F1 treated), it was ineffective in influencing the properties or the deformation modes. Consequently, when buckling of the tubes occurred, the walls still pulled away from the foam and no dilation or lateral strain occurred in the foam. This effect was common to all the tubes and is illustrated in Fig. 3 which is a longitudinal section through an F1 aluminum tube showing that the foam fails to follow the folds during axial crushing.

Dynamically tested transverse samples showed the same general trends of increased flow stresses for the foam-filled tubes. Fig. 4 shows specific energy data for AR and F1 brass tubes and it should be noted that, again, foam-filled tubes performed even better than as-received tubes. Data for the AR tube is unreliable especially at
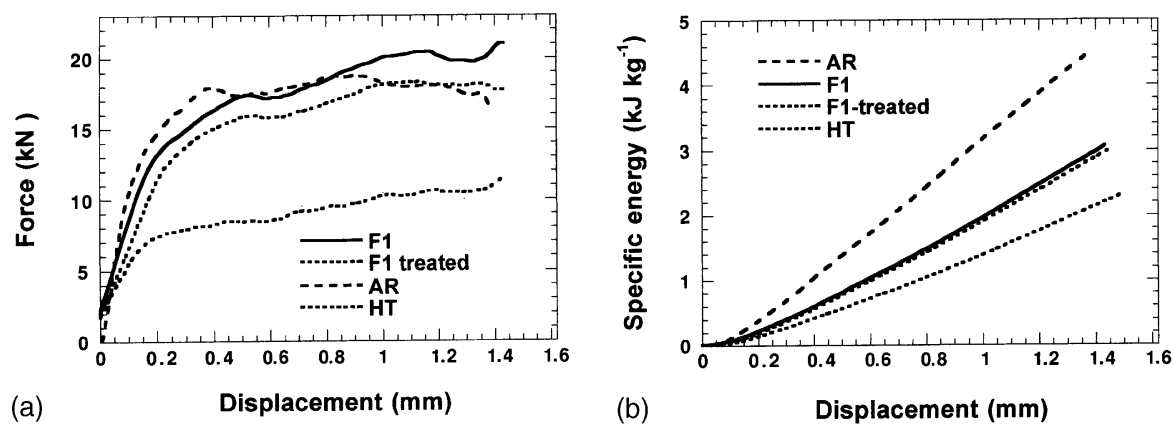

Fig. 2. (a) Dynamic force vs. displacement data for axially loaded brass tube samples. (b) Specific energy absorption vs. displacement for brass tubes. 


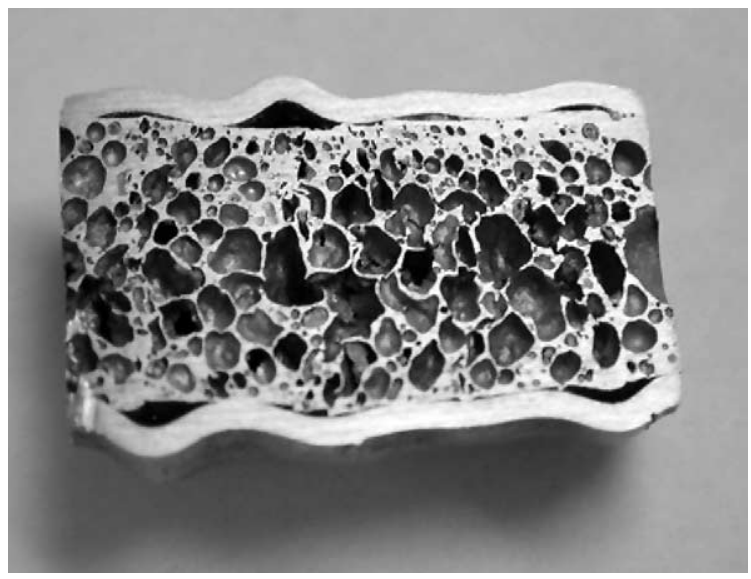

Fig. 3. Section through an F1 Al tube showing tube walls pulling away from foam.

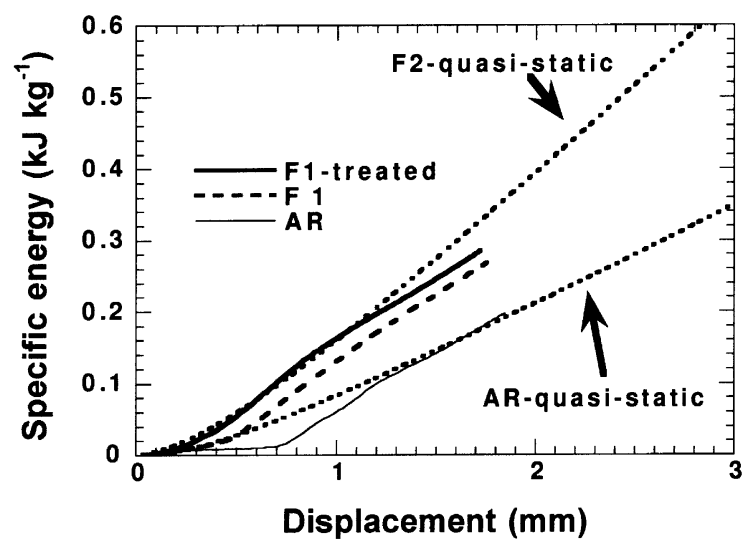

Fig. 4. Specific energy data for dynamically tested AR and F1 brass tubes (quasi-static data shown for comparison). very small displacements since the transmitted wave was too small for accurate load calculations: also, since the yield strength of HT tubes was even further reduced, empty heat treated tubes yielded no useful test data. Note also, surface treatment had no effect on the specific energy absorption, as previously noted for the axial direction.

Upon examination of the tested samples, the most immediately notable feature of transversely tested samples was that, in contrast to axially deformed samples, the foam filling exhibited considerable lateral strain during deformation. As a consequence, the foam remained in contact with the side walls of the tubes throughout the test, and appeared to exhibit a clear, non-zero Poisson's ratio: this phenomenon will be considered further in the discussion section below. It was also noted that the foam would often tear in two along the centerline during the last stages of deformation. Fig. 5(a) is a section through a transversely tested foam-filled titanium tube showing that the foam follows the contours of the tube as it is crushed. Closer examination also showed that the deformation mode of the tubes had been fundamentally altered by the presence of the foam and this is seen by comparing the filled tube with an empty $\mathrm{Ti}$ tube, Fig. 5(b). The empty tube is seen to comprise essentially four quadrants separated by four plastic hinge points as indicated, each quadrant being a $90^{\circ}$ segment of a circle. However, the filled tube exhibited less well-developed hinge points and closer examination shows that the four quadrants were elliptical rather than circular segments. (a)

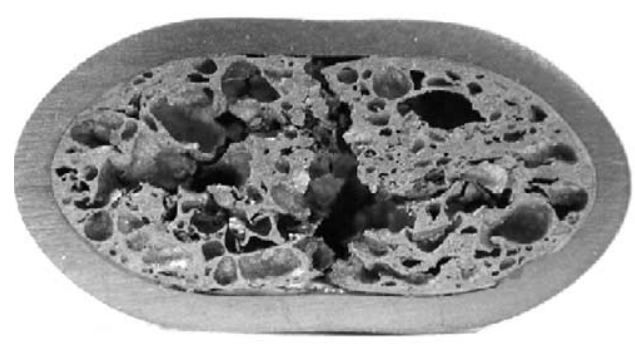

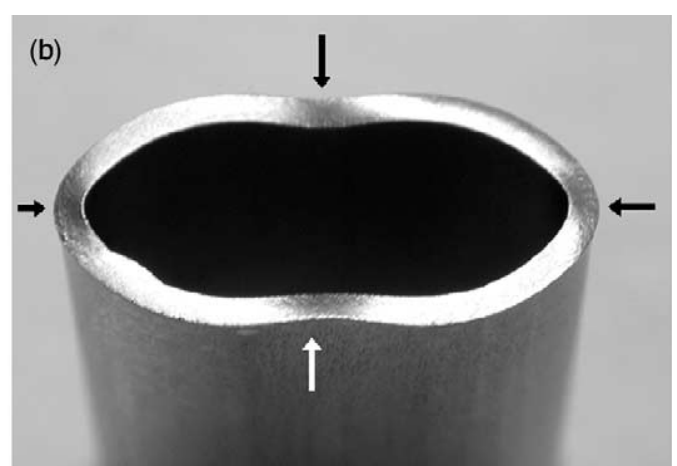

Fig. 5. (a) Section through a transversely tested foam-filled Ti tube. (b) Empty Ti tube showing the four quadrants separated by four plastic hinge points. 


\section{Discussion}

The present results confirm that the advantage of foam-filling, that has already been demonstrated in the axial direction, persists in the transverse direction as well. In general, the specific energy absorption characteristics are significantly less in the transverse direction than in the axial direction as would be expected but they are nevertheless appreciable. In fact, in percentage terms, the improvement in the transverse direction can greatly exceed that in the longitudinal direction. This is most clear for the aluminum tubes. Comparison with the denser brass tube of the same dimensions shows that the latter benefits by approximately half of this amount.

It is reasonable to question whether the advantage gained by foam-filling is greater than that which could be obtained by simply using a thicker tube. The transverse crushing load of a thin walled tube is given, for small deflections, by [10],

$P=\frac{\sigma_{0} t^{2} L}{R \sqrt{1-\left(\frac{\delta}{D}\right)^{2}}}$

where $P$ is crushing load, $\sigma_{0}$ is the yield strength of the tube material, $\delta$ is displacement and $t, R, D$ and $L$ are the thickness, mean radius, mean diameter and length of the tube, respectively. Eq. (1) was developed assuming an elastic/perfectly plastic material and, therefore, only the quasi-static load values corresponding to small displacements, 1-2 $\mathrm{mm}$, were fitted to it in order to predict initial crushing load marked as $P_{0}$ in Fig. 6 and corresponding $\sigma_{0}$ values of each tube material. The fitting results are shown in Fig. 6 and give $\sigma_{0}$ values (124, 475 and $600 \mathrm{MPa}$ for Al, Brass and Ti respectively) that are comparable with the measured ones (145 and $400 \mathrm{MPa}$ for Al and brass [11,12]). A further calculation has been made as follows: using Eq. (1) and the calculated $\sigma_{0}$ values, the thickness of the empty tube which would give the same $P_{0}$ as filled tube at $1 \mathrm{~mm}$ displacement was calculated for each tube. The required thickness of such a thicker tube would be 1.8, 1.17 and 1.071 $\mathrm{mm}$ for Al, brass and Ti tubes, respectively. These corresponding, empty tubes would weigh $1.54,1.2$ and 1.1 times more than the filled tubes for $\mathrm{Al}$,

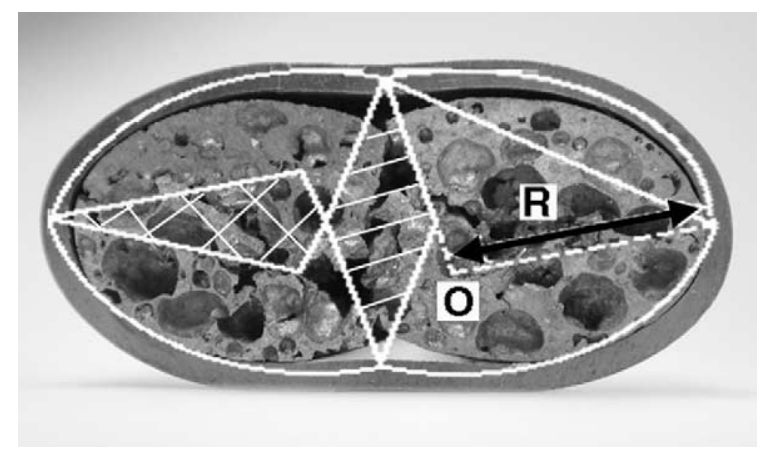

Fig. 6. Calculated and measured crushing forces for all tubes.

brass and $\mathrm{Ti}$, respectively. Although the above analysis is only a approximation, it clearly shows that foam filling can provide a weight-saving advantage also in the transverse direction. The situation might be somewhat different, however, at higher deflections where the foam begins to densify and the specific force vs. deflection curve begins to increase rapidly.

A further major observation is that, while axial crushing of filled tubes leads to negligible transverse strain in the foam, transverse crushing seems to lead to the appearance of considerable transverse strain in it. This may be explained as follows after the treatment of Johnson [13] and is illustrated in Fig. 7 which shows a schematic diagram of the mechanism overlaid on a micrograph of a foam-filled brass tube after testing. At small

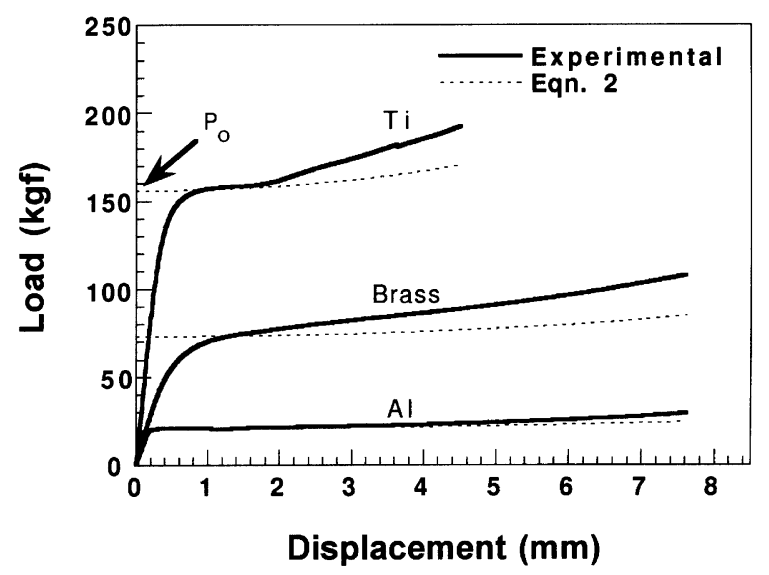

Fig. 7. Tested foam-filled brass sample and schematic diagram indicating deformation mode. 
deflections, a transversely crushed tube develops four plastic hinges and each quadrant rotates independently about its center of curvature, $O$, as shown schematically for the upper right quadrant in Fig. 7. Rotation of the four centers of curvature causes a tensile tearing of the foam in the hatched region (vertically in the center) and a tendency for it to be crushed along the horizontal region (one of which is shown cross-hatched), Fig. 7. The consequence of this is that, theoretically, each of the foam segments may rotate until its maximum dimension is horizontal and, during this time, the foam continues to contribute significantly to energy absorption. The maximum quadrant dimension is the chord shown in Fig. 7. It is clear, therefore, that the apparent non-zero Poisson's ratio noted above is a consequence of the deformation mode of the tube. Furthermore, the lateral spreading of the foam, Eq. (2), is limited to twice the chord length,

$X=\frac{2 R}{\cos 45^{\circ}}$

or $\sim 2.8$ times the tube radius. More severely deformed tubes do indeed show the foam pulling away from the tube walls at the horizontal edges.

\section{Conclusions}

The advantages of foam filling of tubes persist also in the transverse direction at quasi-static and high strain rate $\left(\sim 650 \mathrm{~s}^{-1}\right)$. The percentage in- crease in specific energy was found to be greater than in the axial direction and was highest in the lightest $\mathrm{Al}$ tube and moderate for denser brass and $\mathrm{Ti}$ tubes. Estimation based on initial crushing loads has shown that, for comparable energy absorption, filled tubes would be lighter than thicker tubes. Unlike axial loading, transverse loading leads to moderate lateral displacement of the foam.

\section{References}

[1] Gibson LJ, Ashby MF. Cellular solids: structure and properties. New York: Cambridge University Press; 1997.

[2] Evans AG, Hutchinson JW, Ashby MF. Prog Mat Sci 1998;43:171.

[3] Santosa S, Wierzbicki TJ. Mech Phys Sol 1998;46:645.

[4] Seitzberger M, Rammerstorfer FG, Degischer HP, Gradinger R. Acta Mechanica 1997;125:93.

[5] Seitzberger M, Rammerstorfer FG, Gradinger HR, Degischer HP, Blaimschein M, Walch C. Int J Solids Structures 2000;37:4125.

[6] Hanssen AG, Langseth M, Hopperstad OS. Int J Solids Structures 2000;24:347.

[7] Hanssen AG, Langseth M, Hopperstad OS. Int J Solids Structures 2000;24:475.

[8] Hall IW, Ebil O, Guden M, Yu C-J. J Mater Sci 2001;36:5853.

[9] Hall IW, Guden M, Yu C-J. Scripta Mater 2000;43:515.

[10] DeRuntz JA, Hodge PG. J Appl Mech 1963;30:391.

[11] Van Horn KR. Aluminum. Ohio: ASM; 1967, p. 313.

[12] Parker ER. Materials Data Book. McGraw-Hill; 1967. p. 124.

[13] Johnson W. Impact Strength of Materials. London: Edward Arnold; 1972. 